EMAS als zentrale Entwicklungsperspektive für ökologisches Wirtschaften

\section{Weitere Verbreitung erforderlich}

\author{
Eine nachhaltig zukunftsverträgliche Entwicklung ist als Leitbild ökologischen \\ Wirtschaftens heute allgemein anerkannt. Strittig diskutiert wird jedoch die \\ Umsetzung dieses Leitbildes in praktisches Handeln. Eine Anwendung des \\ EG-Öko-Audit-Systems auf breiter Basis kann hierzu einen sinnvollen Beitrag \\ leisten.
}

$0^{2}$

Von Adolf von Röpenack nung (EMAS) Vorgaben für die freiwillige Einführung eines Umweltmanagementsystems in der gewerblichen Wirtschaft. Damit wurden erstmals die Vorstellungen und Ideen des Leitbildes einer nachhaltigen Entwicklung mit den handelnden Personen in Unternehmen in Verbindung gebracht. Bereits die Enquete-Kommission des zwölften Deutschen Bundestages „Schutz des Menschen und der Umwelt“ hat diesen Schritt als die neue Ethik der Wirtschaft bezeichnet und damit der Einfuihrung eines Umweltmanagementsystems einen hohen moralischen Wert beigemessen (1).

Die Ansprüche, die damit an ein Umweltmanagementsystem gestellt werden, sind entsprechend hoch und es muß gefragt werden, in welcher Wirtschaftsordnung diese überhaupt erfïllt werden können. Sozialistische oder kommuni-

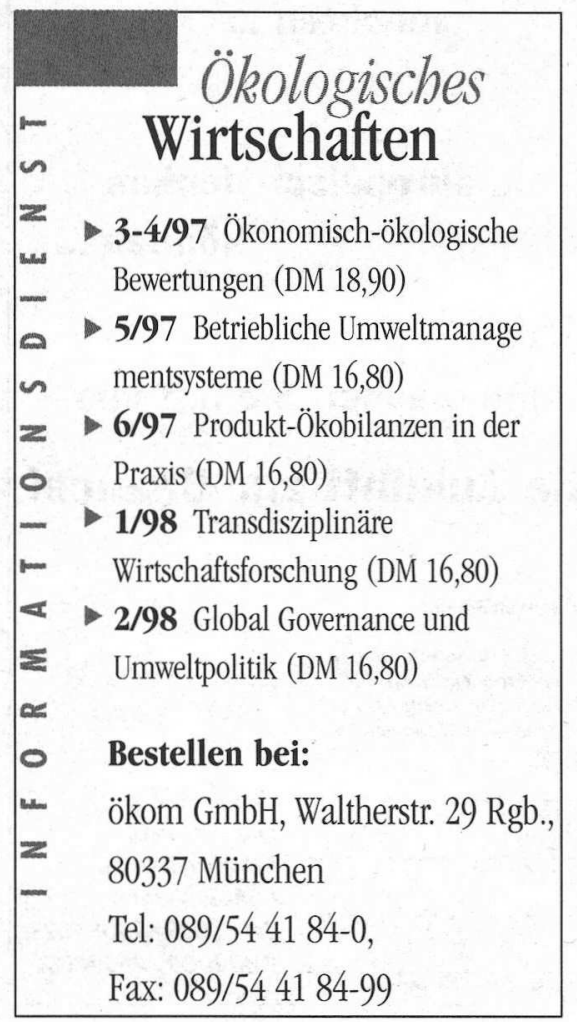

stische Wirtschaftsordnungen scheiden aus, da sie sich als unfähig erwiesen haben, die Bedürfnisse des Menschen zu erfüllen. Es verbleibt damit nur die Marktwirtschaft. Die Anforderungen an ein Umweltmanagementsystem decken sich in bestimmten Teilen mit den Kriterien Ludwig Erhards für die soziale Marktwirtschaft. Diese basiert auf Effizienz und Leistungswettbewerb. Angedacht war in dieser Marktordnung auch eine Wertegesellschaft im Sinne sozialer Verantwortung. Der Umweltschutz wurde als integraler Bestandteil betrachtet. Generell gilt Eigenverantwortung statt staatliches Handeln. Die Wirtschaftsordnung unseres Landes basiert auf diesen Prinzipien und bietet daher gute Voraussetzungen für die Einführung von EMAS.

\section{Grundprinzip Freiwilligkeif}

Mit der Einführung eines Umweltmanagementsystems soll der Umweltschutz in alle Aktivitäten eines Unternehmens und in sein wirtschaftliches Handeln ohne Ausnahmen eingeführt werden. Dieses gilt für den Umweltverbrauch in der Produktion wie beim Design seiner Produkte. Auch das Verhalten der Lieferanten, Kunden und Mitarbeiter ist einzuschließen. Der Umweltschutz beginnt also bereits vor dem Werktor und endet weit danach, wenn auch der Standort des Unternehmens im Zentrum des Handelns steht. Im eigenen Bereich kann das Unternehmen logischerweise die Anforderungen voll durchsetzen, während die Beeinflussung externer Partner nur begrenzt möglich sein und stark von der Marktposition des Unternehmens abhängen dürfte.

Das Umweltmanagementsystem umfaßt alle Ebenen der Organisation des Unternehmens und ist damit ein integraler Teil seines Gesamtmanagements. Mit der systematischen und vollständigen Bestandsaufnahme umweltbezogenen Verhaltens durch eine Umweltprïfung oder eine Umweltbetriebsprüfung ist eine Verpflichtung verbunden, die Umweltleistung des Unternehmens kontinu- ierlich zu verbessern. Dabei bilden nationale und europäische gesetzliche Normen und Standards den Ausgangspunkt.

Die Unternehmen übernehmen mit der Einführung von EMAS eine weitgehende Selbstregulierung im Umweltschutz aus eigener Erkenntnis und Verantwortung heraus. Diese Freiwilligkeit des Handelns wird dem ethischen Ansatz gerecht und ist die Basis für langfristigen Erfolg, der durch das Ordnungsrecht allein nicht erreicht werden kann.

\section{Glaubwürdigkeit ist zentral}

Ein zweiter zu betrachtender Aspekt ist die Glaubwürdigkeit der durchgeführten Maßnahmen. Nur die höchste Managementebene eines Unternehmens kann derart weitgehende Verpflichtungen übernehmen, im Unternehmen durchsetzen, organisatorische Änderungen veranlassen und die Verantwortung auch für möglicherweise unproduktive Kosten übernehmen. Nur dort kann auch die entscheidende Verantwortung angesiedelt sein, beispielsweise gegenüber dem Aufsichtsrat, den Aktionären oder Eigentümern. Das Management muß also die erforderliche Legitimität besitzen, eigenverantwortlich handeln zu können.

Diese hohe Bedeutung des Managements im Rahmen von EMAS ändert sich auch nicht durch die Tatsache, daß der Erfolg von EMAS sehr stark von der Mitarbeit und dem Engagement der Mitarbeiter abhängt. Das Management hat sogar die zusätzliche Aufgabe, seine Mitarbeiter dazu auszubilden und für umweltbewußtes Handeln zu motivieren.

Nötig ist jedoch auch Glaubwürdigkeit gegenüber allen Beteiligten innerhalb und außerhalb des Unternehmens, gegenüber Interessengruppen im weitesten Sinne und allen weiteren Stakeholdern. Daher muß über die Umweltleistung des Unternehmens eine glaubwürdige und transparente Berichterstattung durch das Management erfolgen.

EMAS erfordert daher mit Recht die Umwelterklärung als die geeignete Form einer derartigen Berichterstattung. Es soll damit ein Dialog mit den Stakeholdern und der Öffentlichkeit gefuihrt werden. Die Umwelterklärung soll inhaltlich die Umweltleistung des Unternehmens und seine Umweltschutzorganisation umfassend und in verständlicher Form beschreiben. Das Management muß in dieser Erklärung zu allen in der Verordnung aufgefiuhrten Aspekten die Unternehmenssituation darlegen und weitere Ziele 
vorstellen. Auch Mißerfolge sind zu kommentieren. Die Umwelterklärung enthält also auch eine Erfolgsbeschreibung früherer Ziele und deren Umsetzungsmaßnahmen.

Wenn das bei der Mehrzahl der bereits registrierten Unternehmen noch nicht ausreichend gelungen ist, so ist $\mathrm{zu}$ hoffen, daß dieser Anspruch bei der Novellierung der Verordnung zwingender und deutlicher formuliert wird. Die Vorgabe und Veröffentlichung der Anforderungen durch den Gesetzgeber erhöht zugleich die Glaubwürdigkeit der Umwelterklärung.

Die Umwelterklärung und die gesamte Umsetzung von EMAS im Unternehmen muß weiter durch einen staatlich anerkannten, unabhängigen Umweltgutachter geprift und gegengezeichnet werden.

Die Teilnahme an EMAS wird durch die Aufnahme des Unternehmens in eine periodisch von der EU veröffentlichte Liste dokumentiert. Eine Streichung von dieser Liste dürfte sicher Wettbewerbsnachteile für das Unternehmen bedeuten. Ein weiterer wichtiger Teil des Systems ist die periodisch $\mathrm{zu}$ wiederholende Umweltbetriebsprüfung, mit der eine Erfolgskontrolle der bislang erreichten Verbesserung der Umweltleistung dokumentiert werden soll.

Erfolge wurden bislang vornehmlich bei der Senkung des Energie - und Rohstoffverbrauchs und im Abfallbereich erreicht. Diese Bereiche müssen jedoch im Laufe der Zeit durch Aktivitäten vertieft werden, wie sie durch die vier bekannten Regeln der Nachhaltigkeit des Wirtschaftens vorgegeben sind. Die Enquete-Kommission hat sie in ihrem Abschlußbericht in großer Klarheit formuliert. Diese vier Regeln beschreiben den verantwortlichen Umgang mit erneuerbaren und nicht-erneuerbaren Ressourcen, die Belastbarkeitsgrenzen der Umweltmedien und enthalten Aussagen über die Zeitmaße anthropogener Eingriffe und natürlicher reaktiver Prozesse (1).

Die geeignete Vorgabe für die Weiterentwicklung der Produktionsprozesse ist durch die Ausrichtung auf die jeweils am besten geeignete und verfiigbare Technik bereits in der Verordnung gemacht worden. Hiermit wird die Suche nach diesen Prozessen vorgeschrieben, mit denen für die jeweilige Aufgabe ein Optimum an Umweltschutz erreicht werden kann. Dieses ist eine sehr dynamische Vorgabe, womit die Kraft der Innovation in den Dienst des Umweltschutzes gestellt werden soll. Auch hierzu hat die Enquete-Kommission strategische Handlungsansätze formuliert, die u.a. die Konzeption des produktions- und produktintegrierten Umweltschutzes, den Grundsatz des ökologischen Designs, die Orientierung an der Natur, Kreislaufwirtschaft, recyclinggerechte Produktion und Produkte, sowie Produktverantwortung umfassen.

Ohne diesen Ansatz der am besten geeigneten Technik ist das Ziel der Nachhaltigkeit nicht erreichbar und damit der große Wert von EMAS zumindest verwässert.

\section{Breitere Beteiligung notwendig}

Das Umweltmanagementsystem nach der EGÖko-Audit-Verordnung ist also sehr anspruchsvoll und fordert von den Unternehmen erhebliche Leistungen, eine neue Unternehmenskultur, einen Wertewandel. Ökologisches Wirtschaften nach den Kriterien der Nachhaltigkeit ist jedoch von einem einzelnen Unternehmen nicht zu erreichen, sondern es erfordert die Beteiligung möglichst aller Unternehmen. Dabei ist es nicht wichtig, ob die Unternehmen nun groß oder klein sind, zum gewerblichen Bereich oder zu den Dienstleistern zählen.

Auch eine geographische Begrenzung allein auf den Bereich der EU ist kontraproduktiv. Nach der Öffnung des Binnenmarktes steht jetzt die Globalisierung zur Diskussion. Kapital und Produkte, Informationen und Wettbewerb stehen überall zur Verfügung und erfolgen in weltweiter Kooperation. Nachhaltigkeit ist weltweit erforderlich, was uns die Heftigkeit von „El Nino“ erst kürzlich deutlich demonstriert hat. Eine weltweite Ausdehnung von EMAS und eine Teilnahmeberechtigung für alle Unternehmen ist daher unverzichtbar.

Die Unternehmen werden natülich mit unterschiedlicher Intensität an der kontinuierlichen Verbesserung ihrer Umweltleistung arbeiten. Strategische oder wirtschaftliche Gründe mögen dafür die Ursache sein. Entscheidend ist, daß sie sich nach ihren Möglichkeiten beteiligen. Wir müssen eine Wertegesellschaft werden und damit kommt es auf jeden Einzelnen an. Ökologische Vorreiter allein lösen das Problem der nachhaltigen Entwicklung nicht, was jedoch die Bedeutung ihrer Vorbildfunktion nicht mindert. Hierzu gehören neben den ökologischen auch noch ökonomische und soziale Aspekte. Erst diese drei Säulen bilden die tragfähige Basis der Nachhaltigkeit. Daher muß der Breite der Beteiligung der Vorzug gegeben werden.

Diese breite Beteiligung erfordert automatisch, daß die Forderung nach komplizierten Kennzah- len zur Beschreibung der Umweltleistung nicht zielführend sein kann. So bedeutungsvoll die direkte Verbindung von Betriebserfolgen mit den Hauptumweltproblemen wie Klima- und Ozonfrage, Eutrophierung der Gewässer und Ausdehnung der Wüsten sowie Artenarmut ist; sie ist dennoch ungeeignet, wenn sie nur von einer Minderheit berechnet und dảher genutzt werden kann. Zeit- oder mengenabhängige Kennzahlen kann dagegen jedes Unternehmen erstellen. Es ist Sache des Staates, komplexe Zusammenhänge auf verständliche Anforderungen herunterzubrechen.

Das Umweltmanagement nach den Vorgaben der ISO 14001 stellt ähnliche Ansprüche wie vorstehend durch EMAS beschrieben, wenn auch nicht in gleicher Tiefe. Es ist aber nicht in allen Aspekten verpflichtend und braucht auch nicht alle Aspekte zu umfassen. Insbesondere fehlt die Umwelterklärung und deren Beglaubigung durch einen Umweltgutachter, sowie die öffentliche Registrierung der Teilnahme. Auch dieses Umweltmanagementsystem fördert den Umweltschutz. Es genügt jedoch nicht dem vorstehend beschriebenen Standard für ökologisches Wirtschaften und dem Prinzip der Nachhaltigkeit.

Eine von der Wirtschaft erwartete Deregulierung bestimmter ordnungsrechtlicher Vorgaben, eine Eigenregulierung im Umweltschutz entsprechend dem Umweltpakt Bayern oder der aktuellen diesbezüglichen Beschlußvorlage für die Umweltminister-Konferenz der Länder ist hiermit ebenfalls nicht zu erreichen.

Die diskutierten Anforderungen an ökologisches Wirtschaften sind sicherlich im Rahmen der sozialen Marktwirtschaft am besten erreichbar. Die Ansätze der Enquete-Kommission stellen eine Hilfe für die Unternehmen dar, die ökologisch wirtschaften wollen. EMAS ist dafür eine unverzichtbare Voraussetzung und sollte daher weltweit Anwendung finden.

\section{Anmerkung}

(1) Enquete-Kommission "Schutz des Menschen und der Umwelt" (Hrsg.): Die Industriegesellschaft gestalten. Perspektiven für einen nachhaltigen Umgang mit Stoffströmen, Bonn 1994.

\section{Der Autor}

Dr. Adolf von Röpenack ist Umweltberater.

Kontakt: Hermannstr. 31, 45711 Datteln.

Tel. $02363 / 62749$, Fax 02363/ 72615 . 
(c) 20I0 Authors; licensee IÖW and oekom verlag. This is an article distributed under the terms of the Creative Commons Attribution Non-Commercial No Derivates License (http://creativecommons.org/licenses/by-nc-nd/3.o/), which permits unrestricted use, distribution, and reproduction in any medium, provided the original work is properly cited. 